\title{
Three-dimensional hydrodynamics simulations of shell burning in Si/O-rich layer of pre-collapse massive stars
}

\author{
Takashi Yoshida ${ }^{1, *}$, Tomoya Takiwaki ${ }^{2}$, Kei Kotake ${ }^{3}$, Koh Takahashi ${ }^{4}$, Ko Nakamura ${ }^{3}$, \\ Hideyuki Umeda ${ }^{5}$, David R. Aguilera-Dena ${ }^{6}$, and Norbert Langer ${ }^{7,8}$ \\ ${ }^{1}$ Yukawa Institute for Theoretical Physics, Kyoto University, Kyoto 606-8502, Japan \\ ${ }^{2}$ Division of Science, National Astronomical Observatory of Japan, Tokyo 181-8588, Japan \\ ${ }^{3}$ Department of Applied Physics \& Research Institute of Stellar Explosive Phenomena, Fukuoka Uni- \\ versity, Fukuoka 814-0180, Japan \\ ${ }^{4}$ Max-Planck-Institute for Gravitational Physics, D-14476 Potsdam, Germany \\ ${ }^{5}$ Department of Astronomy, Graduate School of Science, The University of Tokyo, Tokyo 113-0033, \\ Japan \\ ${ }^{6}$ Institute of Astrophysics, FORTH, Department of Physics, University of Crete, GR-71003 Heraklion, \\ Greece \\ ${ }^{7}$ Argelander-Institut für Astronomie, Universität Bonn, D-53121 Bonn, Germany \\ ${ }^{8}$ Max-Planck-Institute für Radioastronomie, D-53121 Bonn, Germany
}

\begin{abstract}
We perform three-dimensional (3D) hydrodynamics simulations of shell burning in the silicon- and oxygen-rich layers in pre-collapse massive stars. We adopt a non-rotating $27 M_{\odot}$ star having an extended $\mathrm{O} / \mathrm{Si} / \mathrm{Ne}$ layer and a fast-rotating $38 M_{\odot}$ star having a $\mathrm{Si} / \mathrm{O}$ layer, that has experienced chemically homogeneous evolution. Both pre-collapse stars show large-scale turbulent motion with a maximum Mach number of $\sim 0.1$ in the convective layers activated by neon and oxygen shell burning. The radial proflie of the angle-averaged mass fraction distribution in $\mathrm{O} / \mathrm{Si} / \mathrm{Ne}$ layer is more homogeneous in the $3 \mathrm{D}$ simulation compared to the $1 \mathrm{D}$ evolution for the $27 M_{\odot}$ star. The angle-averaged specific angular momentum in the $\mathrm{Si} / \mathrm{O}$ layer of the fast-rotating $38 M_{\odot}$ star tends to become roughly constant in the convective layer of the $3 \mathrm{D}$ simulation, which is not considered in the $1 \mathrm{D}$ evolution.
\end{abstract}

\section{Introduction}

Multi-dimensional (multi-D) hydrodynamics simulations of shell burning in convective layers during advanced evolution of massive stars have been performed to investigate the convection properties in evolved massive stars (e.g., [1,2]). Recently, it has been pointed out that asphericity of wide silicon- and oxygen-rich $(\mathrm{Si} / \mathrm{O})$ convective layers, such as large-scale convective flows, in pre-collapse stars gives a favorable condition to energetic supernova explosion $[3,4]$. Then, three-dimensional (3D) hydrodynamics simulations of the Si/O convective layers of pre-collapse massive stars have been performed for hundreds seconds and showed a large-scale and high-velocity turbulent motion in these layers (e.g., [5-8]). We perform 3D hydrodynamics simulations of shell burning in wide $\mathrm{Si} / \mathrm{O}$-rich convective layers of

\footnotetext{
*e-mail: yoshida@yukawa.kyoto-u.ac.jp
} 

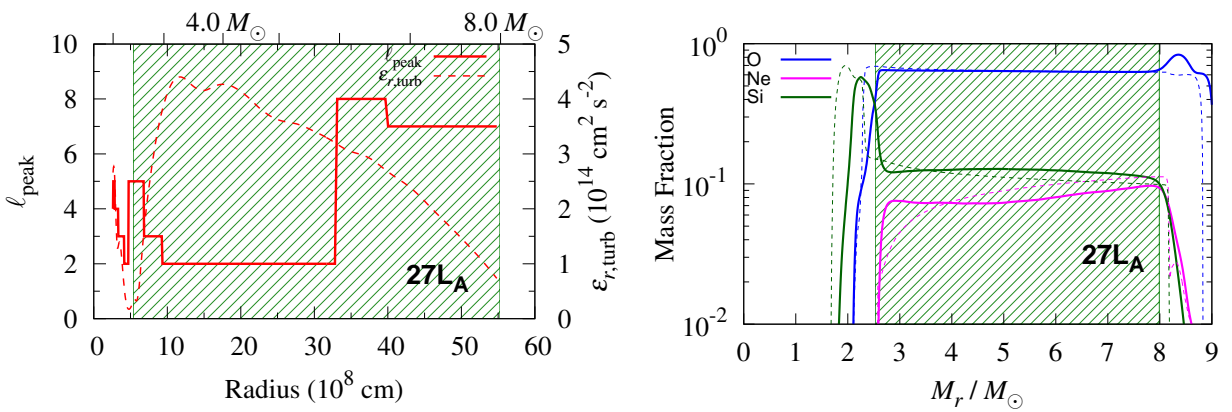

Figure 1. Left panel: The radial profiles of the peak mode $\ell_{\text {peak }}$ obtained by the spectrum analysis of the radial turbulent velocity (solid curve) and the radial turbulent kinetic energy (dashed curve) of the $27 M_{\odot}$ star. Right panel: the radial profiles of the oxygen (blue), neon (green), and silicon (magenta) mass fractions. Solid and dashed curves correspond to the last and initial profiles of the 3D simulation.

pre-collapse massive stars for 100-200 s. We investigate convection properties of the $\mathrm{O} / \mathrm{Si} / \mathrm{Ne}$ layer induced by the neon shell burning in a non-rotating $27 M_{\odot}$ star and rotation effects of the oxygen-shell burning in the $\mathrm{Si} / \mathrm{O}$ layer of a fast-rotating $38 M_{\odot}$ star.

\section{Models}

We adopt two pre-collapse massive star models for 3D hydrodynamics simulations of shell burning. The one is a non-rotating $27 M_{\odot}$ star (model 27 $\mathrm{L}_{\mathrm{A}}[7,9]$ ) with the solar metallicity, having the convective $\mathrm{O} / \mathrm{Si} / \mathrm{Ne}$ layer with the range of $(5.8-60) \times 10^{8} \mathrm{~cm}$. The other is a fastrotating $38 M_{\odot}$ star with $Z \sim 1 / 50 Z_{\odot}$ and the initial rotation velocity of $600 \mathrm{~km} \mathrm{~s}^{-1}[10,11]$, having the $\mathrm{Si} / \mathrm{O}$ convective layer in the range of $(4.7-17) \times 10^{8} \mathrm{~cm}$. This star has experienced chemically homogeneous evolution. We perform 3D hydrodynamics simulations of the 27 $M_{\odot}$ star and the fast-rotating $38 M_{\odot}$ star for $218.5 \mathrm{~s}$ and $91.6 \mathrm{~s}$, respectively, until the central temperature becomes $10^{10} \mathrm{~K}$ using multi-dimensional hydrodynamics code $3 \mathrm{DnSEV}$ [7].

\section{Results}

First, we show convection properties of the neon shell burning in the $\mathrm{O} / \mathrm{Si} / \mathrm{Ne}$ layer of the 27 $M_{\odot}$ star [9]. We obtained the angle-averaged turbulent Mach number of $\left\langle M a^{2}\right\rangle^{1 / 2} \sim 0.1$. The angle-averaged turbulent velocity reaches $4.2 \times 10^{7} \mathrm{~cm} \mathrm{~s}^{-1}$ (see also left panel of Figure 1). This high-velocity turbulence forms large-scale turbulence eddies in the convective layer. In the power spectrum analysis of the radial turbulent velocity, the peak mode of $\ell_{\text {peak }}=3$ is obtained in a wide range of the convective layer (left panel of Figure 1). The averaged value of the peak mode in the $\mathrm{O} / \mathrm{Si} / \mathrm{Ne}$ layer is $\left\langle\ell_{\text {peak }}\right\rangle=3.56$. The turbulent motion is activated by neon shell burning during the contraction and extends to the outer boundary.

The turbulent mixing in the 3D simulation causes the radial profile of the angle-averaged neon and silicon mass fractions different from that in the 1D stellar evolution. The radial gradient of the neon and silicon mass fractions in the $\mathrm{O} / \mathrm{Si} / \mathrm{Ne}$ layer in the $3 \mathrm{D}$ simulation becomes less steep compared to the 1D evolution profile (right panel of Figure 1). The mixing time scale of the 3D simulation is compatible to the advection time scale of the turbulent flow and shorter than the diffusion time scale deduced using the mixing length theory. 

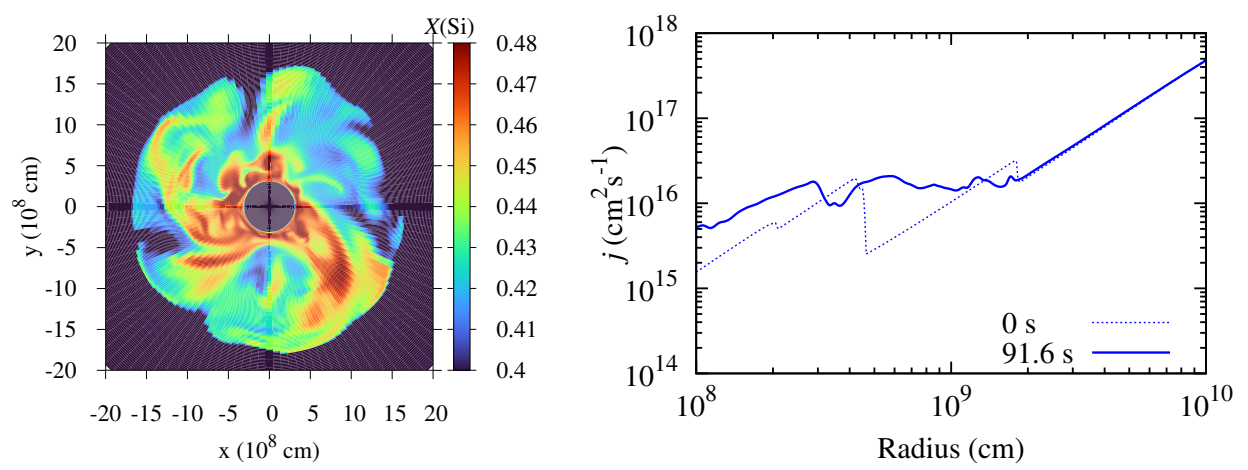

Figure 2. Left panel: Slices in the $x-y$ plane showing the silicon mass fraction distribution at the last time step (91.6 s) of the fast-rotating $38 M_{\odot}$ star. Right panel: Radial profiles of the angle-averaged specific angular momentum at the initial (dotted curve) and last (solid curve) time steps.

Next, we show convection and rotation properties of the oxygen shell burning in the $\mathrm{Si} / \mathrm{O}$ convective layer of the fast-rotating $38 M_{\odot}$ star. This star also shows a large-scale turbulence with high turbulent velocity. The angle-averaged turbulent Mach number becomes $\left\langle M a^{2}\right\rangle^{1 / 2} \sim 0.135$ at the maximum. The power spectrum analysis of the radial turbulent velocity indicates the peak mode of $\ell=3$ in the range of $(5-13) \times 10^{8} \mathrm{~cm}$. Spiral-arm structures of silicon enhancement in the silicon mass fraction distribution in the equatorial plane are seen as a rotation effect in this layer (left panel of Figure 2). We also obtain dominant low order modes $(m \sim 1-3)$ in the fluctuations of the density and rotational kinetic energy density.

The angular momentum transfer in the $\mathrm{Si} / \mathrm{O}$ convective layer is investigated for the fastrotating star. We see that the the monotonically increasing profile of the angle-averaged specific angular momentum in the $\mathrm{Si} / \mathrm{O}$ layer, corresponding to a rigid rotation, changes to a roughly constant profile in the 3D simulation (right panel of Figure 2). This profile is different from the convective layer considered in 1D spherically symmetrical evolution models. To confirm such kind of angular momentum transfer by convection, we would need to investigate for longer time scale achieving a steady state convection.

\section{References}

[1] G. Bazán, D. Arnett, Astrophys. J. 496, 316 (1994)

[2] C. A. Meakin, D. Arnett, Astrophys. J. Lett. 637, L53 (2006)

[3] S. M. Couch, C. D. Ott, Astrophys. J. Lett. 778, L7 (2013)

[4] B. Müller, H.-T. Janka, Mon. Not. R. Astron. Soc. 448, 2141 (2015)

[5] S. M. Couch, et al., Astrophys. J. Lett. 808, L21 (2015)

[6] B. Müller et al., Astrophys. J. 833, 124 (2016)

[7] T. Yoshida et al., Astrophys. J. 881, 16 (2019)

[8] N. Yadav, et al., Astrophys. J. 890, 94 (2020)

[9] T. Yoshida, et al., Astrophys. J. 908, 44 (2021)

[10] D. R. Aguilera-Dena, et al., Astrophys. J. 901, 114 (2020)

[11] T. Yoshida, et al., Mon. Not. R. Astron. Soc. Lett. 506, L20 (2021) 